\title{
Systematic rapid living review of the impact of the COVID-19 pandemic on cancer survivors: update to August 27, 2020
}

\author{
Anish Singh Jammu ${ }^{1} \cdot$ Martin Robert Chasen ${ }^{2,3,4,5}$ (I) Aisha Kamilah Lofters ${ }^{3,6,7,8,9,10} \cdot$ Ravi Bhargava ${ }^{5,11}$
}

Received: 14 September 2020 / Accepted: 20 November 2020 / Published online: 26 November 2020

(C) Springer-Verlag GmbH Germany, part of Springer Nature 2020

\begin{abstract}
Background The COVID-19 pandemic has resulted in drastic changes in the global healthcare delivery landscape and has had practical repercussions for cancer survivors. This systematic rapid living review has been undertaken to synthesise the available knowledge regarding the impact of the COVID-19 pandemic in a timely manner. This initial rapid review will present the findings of literature published up to August 27, 2020.

Design A systematic search of PubMed, Scopus and Google Scholar databases was conducted to identify all articles, available in English language, regarding the impact of the COVID-19 pandemic on cancer survivors published between December 2019 and August 27, 2020. The search strategy employed the following search strings: "covid-19 OR coronavirus OR sars-cov-2" with "cancer survivors OR cancer survivorship".

Results The database search yielded 1639 articles, of which 19 were included. Of the 19 selected articles, there were 12 expert opinion articles, two literature reviews, two prospective cohort studies, one retrospective cohort study, one descriptive study and one pooled meta-analysis that comment on the impact of the COVID-19 pandemic on the physical wellbeing (16 articles), psychosocial wellbeing (15 articles) and financial wellbeing of cancer survivors (3 articles).

Conclusions Limited definitive evidence exists regarding the impact of the COVID-19 pandemic on cancer survivors. Currently available literature provides preliminary indications of wide-ranging impacts of the pandemic on cancer survivors with respect to the requirement to adapt to new means of healthcare delivery as well as their physical, psychosocial and economic wellbeing. The pandemic has left survivors dealing with the consequences of rigorous cancer treatment in the context of new challenges related to social isolation, financial hardship and uncertainty with respect to their ongoing care. Additional rigorously designed research initiatives are required to elucidate the impact of the pandemic on cancer survivors.
\end{abstract}

Keywords Coronavirus $\cdot$ Severe acute respiratory syndrome $\cdot$ SARS-CoV-2 COVID-19 · Cancer survivorship · Cancer survivors

Martin Robert Chasen

martin.chasen@williamoslerhs.ca

1 Global Health Program, McMaster University, 1280 Main Street West, Hamilton, ON L8S 4L8, Canada

2 Division of Palliative Care, William Osler Health System, 2100 Bovaird Dr E, Brampton, ON L6R 3J7, Canada

3 Department of Family and Community Medicine, University of Toronto, Toronto, ON, Canada

4 Department of Family Medicine, McMaster University, Hamilton, ON, Canada
5 The Global Institute of Psychosocial, Palliative and End-of-Life Care, University of Toronto, Toronto, Ontario, Canada

6 Women's College Hospital, Family Practice Health Centre, Toronto, Ontario, Canada

7 MAP Centre for Urban Health Solutions, Li Ka Shing Knowledge Institute, St. Michael's Hospital, Toronto, ON, Canada

8 Department of Family and Community Medicine, St. Michael's Hospital, Toronto, ON, Canada

9 ICES, Toronto, ON, Canada

10 Dalla Lana School of Public Health, Toronto, ON, Canada

11 PHMI Program Queen's University, Kingston, ON, Canada 


\section{Introduction}

The COVID-19 pandemic, caused by severe acute respiratory syndrome coronavirus 2 (SARS-CoV-2), was first detected in Wuhan, China in December 2019. It quickly escalated to levels that required the World Health Organization (WHO) to declare a pandemic by March 2020 [1]. In a matter of months, this global pandemic has severely impacted healthcare service delivery across the world. In specific, healthcare services associated with non-communicable diseases (NCDs) have been disproportionately and significantly disrupted. A survey of 155 countries, conducted by the WHO, found that $42 \%$ of countries reported disruptions to cancer treatment and an astounding 63\% reported service disruptions in rehabilitation services, which include survivorship care [2].

It is evident that the impact of COVID-19 extends far beyond that of an infectious disease itself [3]. The emergence of this global pandemic has resulted in radical changes in the global healthcare delivery landscape and has had practical repercussions for cancer survivors because essential components of survivorship care (i.e. ongoing oncological screening and surveillance, physical symptom support, psychosocial symptom support and promotion and guidance on healthy lifestyle maintenance) have traditionally been heavily dependent on in-person healthcare interactions [4]. The current circumstances have reduced the accessibility of already limited support services during a time when anxiety, depression and COVID-19-related fears appear to have increased among survivors [5].

New social distancing requirements imposed by governments based on evidence-based international recommendations and the deferral of non-emergent, elective and preventive medical services have detrimentally impacted to state of survivorship care and exposed fragilities in the current delivery of care $[4,6,7]$. In fact, survivorship visits have been placed at lowest priority in some countries despite the fact that experts have stressed the importance of maintaining the emotional wellbeing of patients through psychosocial support during the pandemic $[8,9]$. This has required many survivors, that previously benefited from significant support and guidance from healthcare professionals and experts, to try to selfmanage symptoms. Consequently, cancer survivors in this scenario may feel helpless and neglected as their unique needs are being classified as 'non-urgent' and are not being addressed in the right place, at the right time, in the right amount and in the right manner. Experts have indicated that such circumstances will detrimentally impact the survivorship "experience and the management of cancer-related symptoms, quality of life and survival" [10, 11].

In fact, anecdotal survivor experiences included in the current and developing literature on the topic have already begun to illuminate the untold toll of the pandemic on patients without COVID-19. Anecdotal information from survivors indicates that they value the "opportunity to learn about social and emotional support services" following active cancer treatment; however, the current situation has necessitated brief interactions over the phone that have left some survivors feeling "overwhelmed and confused" and "the lack of face-to-face interaction, compounded by the unavailability of typical support systems has made it difficult to cope" [12]. Experts and healthcare professionals are calling for increased research efforts to comprehensively understand the consequences of the pandemic on cancer survivors [13].

This systematic rapid living review has been undertaken with the goal of synthesizing the available knowledge regarding the impact of the COVID-19 pandemic in a timely manner. This initial rapid review will present the findings of literature published up to August 27, 2020 and will be updated on a regular basis as new and relevant literature on the subject becomes available.

\section{Methods}

\section{Design}

Systematic review completed in accordance with PRISMA guidelines [14].

\section{Search strategy}

On August 27, 2020, a systematic search of PubMed, Scopus and Google Scholar databases was conducted to identify all articles, available in English language, regarding the impact of the COVID-19 pandemic on cancer survivors who have completed primary anti-cancer treatment and are free of disease, published between December 2019 and August 27, 2020. The search strategy employed included the following search strings: "covid-19 OR coronavirus OR sars-cov-2" with "cancer survivors OR cancer survivorship". This study has been submitted to PROSPERO (Registration number: CRD42020197012) and was developed in accordance with the Preferred Reporting Items for Systematic Reviews and Meta-Analysis Protocols (PRISMA-P).

\section{Study selection}

One author (A.J.) independently completed all searches using the pre-established search strategy. In order to identify additional articles of relevance, the references of selected articles were hand searched. Duplicate records were systematically identified and removed. Identification of eligible studies took place in two stages.

During the first screening stage, eligible studies were selected by two authors (A.J., M.C.) based on the relevance of study titles and abstracts. In this review, eligible study titles 
and abstracts were required to be available in the English language; however, there were no eligible studies that were removed as a consequence of this restriction. The second screening stage involved a careful review of the full-text articles deemed relevant in the first stage. A critical appraisal of each of the full-text articles was conducted to ensure that the selected articles reported on a relevant topic associated with the impact of COVID-19 on cancer survivors who have completed primary anti-cancer treatment and are free of disease. The reference lists of articles were hand searched in an attempt to identify additional relevant articles. Discrepancies and general queries were resolved through consensus with two more authors (R.B., A.L.).

\section{Data extraction}

One author (A.J.) independently extracted data and information from the eligible studies using a data extraction table generated in Microsoft Excel. Data extracted from the selected articles included (if applicable): (1) article citation; (2) study design; (3) study aim; (4) study/target population; (5) study/ target setting; (6) country of origin and (7) relevant content/ main findings. The quality of the included studies was not appraised.

\section{Analysis}

A narrative synthesis of the results and conclusions of the eligible studies was conducted.

\section{Results}

Of the 1639 articles identified through a database search, 19 were included in the qualitative synthesis. Figure 1 indicates the specific study screening process.

The selected articles present information related to the impact of the COVID-19 pandemic on cancer survivors directly or indirectly. Of the 19 selected articles, there are 12 expert opinions (including editorials, calls to action, clinical correspondence), two literature reviews, two prospective cohort studies, one retrospective cohort study, one descriptive study and one pooled meta-analysis. Overall, 16 of the articles comment on the impact of the COVID-19 pandemic on the physical wellbeing of cancer survivors, 15 address the impact of the pandemic on the psychosocial wellbeing of survivors and three focus on the economic consequences of the COVID-19 pandemic related to the financial wellbeing of survivors. These articles originate from the USA $(n=9)$, USA and Canada $(n=2)$, Hong Kong $(n=2)$, Canada $(n=1)$, Australia $(n=1)$, China $(n=1)$, Sri Lanka $(n=1)$ and multinational collaboration $(n=2)$. Relevant findings and conclusions of each of the 19 articles are contained in Table 1 . These articles have been categorised based on specific content areas related to the impact of the COVID-19 pandemic on cancer survivors. Some of the articles fall into multiple categories as a result of the breadth of information covered in specific publications.

\section{Survivorship care delivery}

The first category is comprised of articles addressing significant changes to the care that survivors receive and the channels through which it is delivered. These articles provide insight into how survivorship care has evolved in response to the new challenges presented by the COVID-19 pandemic and in an attempt to reduce the risk of infection transmission. Calvillo-Argüelles et al. [6] and Dietz et al. [8] point out that the current recommendation based on available evidence is the temporary modification or postponement of routine surveillance and follow-up of asymptomatic cancer survivors. Chan et al. [7] and Koczwara [11] indicate that survivors have seen significant delays and cancellations of follow-up appointments and the replacement of most in person interactions with telehealth. However, Chan et al. [7] have noted that many barriers continue to impede widespread implementation of telehealth, and great disparities exist in how patient needs are being triaged amid the pandemic. Ratnasekra et al. [26] have also highlighted that lockdown restrictions imposed due to COVID-19 have impacted the availability and accessibility or routine healthcare services for cancer patients and survivors. In fact, Papautsky and Hamlish [25] conducted a survey of breast cancer survivors in which $44 \%$ reported delays across all aspects of cancer care and treatment. Flannigan et al. [19], Jhaveri et al. [21] and Nilsen et al. [24] demonstrate how the pandemic has necessitated drastic changes in the delivery of some cancer survivorship programs. While there has been an effort to rapidly transition to delivering elements of survivorship care (e.g. physical activity, nutrition support, stress management and spiritual wellness) through telehealth, some elements of operations have been temporarily suspended, postponed or removed by necessity. As such, the COVID-19 pandemic has drastically reduced the frequency and types of care survivors are able to access as jurisdictions attempt to reduce risk of COVID-19 transmission through inperson healthcare encounters.

\section{Psychosocial and physical wellbeing}

The second group includes articles that comment on the impact of the pandemic on the psychosocial and physical wellbeing of cancer survivors. Boakye et al. [16] and Huang et al. [20] noted that postponement of surveillance and other elective procedures to improve quality of life may lead to increase anxiety, delayed diagnosis of disease recurrence and reduced quality of life. Chan et al. [7] indicated that some 
Fig. 1 PRISMA flow diagram depicting the study screening process

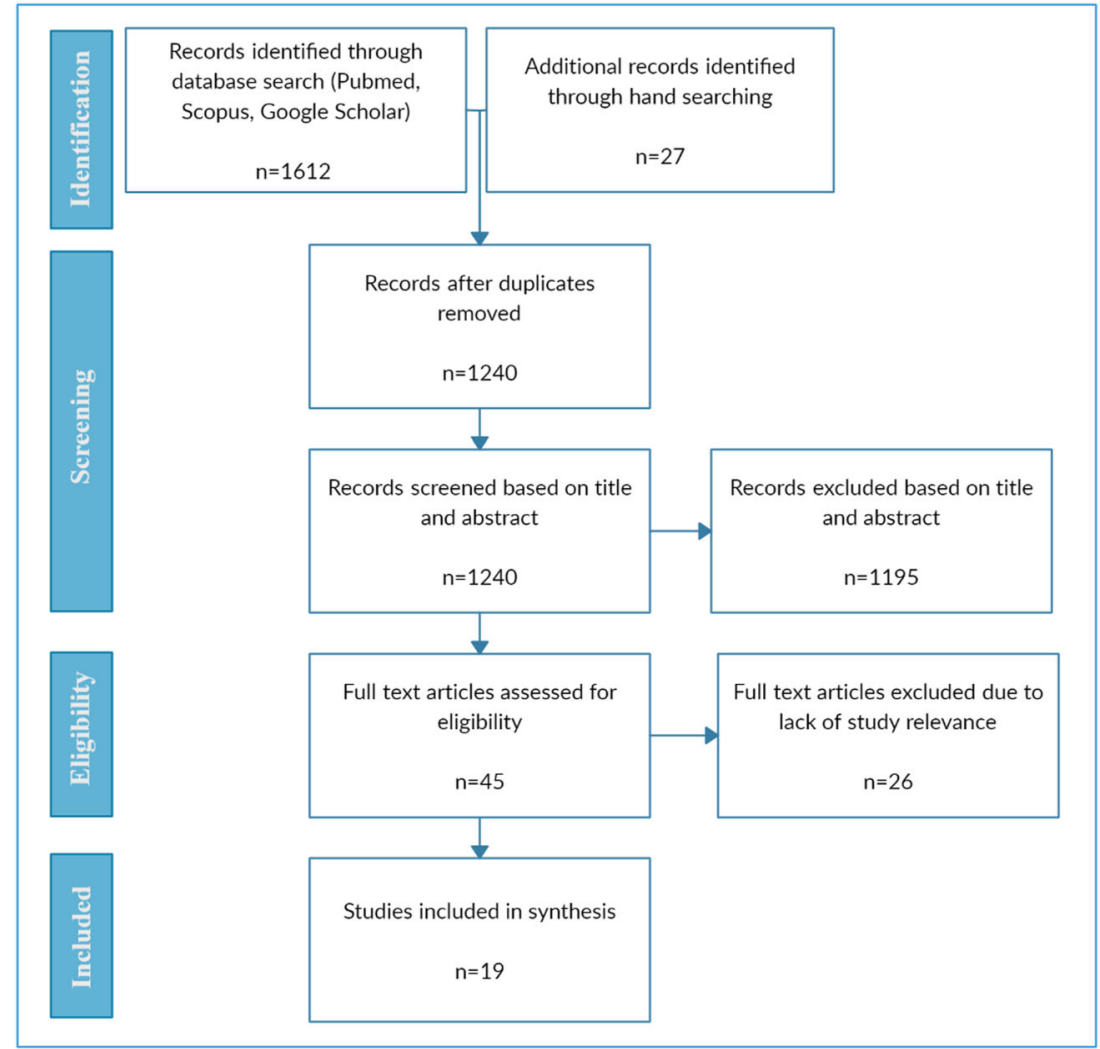

healthcare providers have noted increased anxiety and distress among survivors in relation to using telehealth, and Jhaveri et al. [21] noted that some survivors have expressed an increased need for behavioural health and social support during the pandemic. Nekhlyudov et al. [13] highlighted that the pandemic may disproportionately impact the physical and psychosocial wellbeing of cancer survivors due to the requirement to maintain physical distancing from their social and support networks and increased anxiety related to cancellation, postponement or modification of follow-up visits and cancer surveillance. Notably, $\mathrm{Ng}$ et al. [23] found that cancer survivors demonstrated greater catastrophizing and health anxiety (but less psychological distress) compared to healthy controls. According to Koczwara [11], cancer survivors experience greater psychological distress and difficulty managing physical symptoms than the general public and may be unable to continue to adhere to lifestyle recommendations due to pandemic restrictions that have limited physical examinations and required survivors to self-administer exercise programs previously delivered by healthcare professionals. Ratnasekra et al. [26] noted that oral cancer survivors experiencing pain and difficulty swallowing faced difficulties in accessing routine treatment and medication due to lockdown restrictions. Cheung et al. [17] highlighted that paediatric cancer survivors believed to have increased susceptibility to COVID-19 have found themselves confined to their homes which may result in downstream adverse health outcomes and poor psychological wellbeing. Young et al. [27] pointed out that cancer survivors also may have trouble difficulty determining whether experienced symptoms are the result of COVID-19 or cancer recurrence and whether to report them. A study by Zhou et al. [28] noted reduced insomnia severity among young adult cancer survivors following COVID-19 lockdown restrictions and highlighted the need to identify factors contributing to better sleep and increased anxiety and depression during the pandemic.

A subset of included articles provides preliminary results indicating that cancer patients and survivors may have increased susceptibility to COVID-19 infection. Liang et al. [22] noted that cancer patients and survivorship with current or past cancer diagnosis may be at increased risk of significant morbidity (including ICU admission, requirement for invasive ventilation or death) compared to patients without cancer. A pooled meta-analysis by Desai et al. [18] also found that patients with cancer and cancer survivors were at increased risk of acquiring COVID-19 and although evidence regarding the association between COVID-19 and cancer is unclear, greater precautions to protect such individuals may be warranted. As such, based on the potential increased susceptibility of cancer survivors to COVID-19, necessity of any interventions must be compared to the risk of inadvertent exposure to COVID-19. 
Table 1 Relevant findings and conclusions of the 19 articles included in this rapid review of the impact of COVID-19 on cancer survivors

\begin{tabular}{lll}
\hline Study Study aim & $\begin{array}{l}\text { Type of } \\
\text { publication }\end{array}$ & Country of origin Relevant findings and conclusions
\end{tabular}

\begin{tabular}{lll}
\hline Baddour et al. [15] & $\begin{array}{l}\text { To review the available } \\
\text { literature on financial } \\
\text { toxicity and economic } \\
\text { challenges experienced by } \\
\text { cancer survivors during } \\
\text { COVID-19. }\end{array}$ & $\begin{array}{c}\text { Literature } \\
\text { review }\end{array}$ \\
Boakye et al. [16] & $\begin{array}{l}\text { To examine the impact of the } \\
\text { COVID-19 pandemic on } \\
\text { head and neck cancer } \\
\text { (HNC) survivors. }\end{array}$
\end{tabular}

USA

(

USA

$$
\text { (HNC) survivors. }
$$
Calvillo-Argüelles et al. [6]

Chan et al. [7]

\begin{tabular}{|c|c|}
\hline Cheung et al. [17] & $\begin{array}{l}\text { To communicate health } \\
\text { support provided to } \\
\text { paediatric cancer } \\
\text { survivors and their } \\
\text { families during the } \\
\text { COVID-19 }\end{array}$ \\
\hline
\end{tabular}

Desai et al. [18] To examine the pooled prevalence of cancer among patients with COVID-19.
Expert opinion USA/Canada

Expert opinion

Multinational collaboration

Expert opinion Hong Kong
The COVID-19 pandemic and its associated economic consequences may potentially disproportionately impact cancer survivors financially and subsequently their health-related quality of life and mortality.

Due to the COVID-19 pandemic, many HNC survivors, who require ongoing medical care, will not be able to continue complete cancer surveillance based on their pre-established timelines and will see the postponement of elective procedures aimed at improving quality of life. This may result in increased anxiety, delays in diagnosis of disease recurrence and reduced quality of life. The pandemic may give rise to financial hardship and negatively impact the physical and psychosocial wellbeing of survivors and caregivers. Potential solutions which may mitigate the negative repercussions of the pandemic include scaling-up telemedicine and increased funding allocation for survivors and caregivers.

Recommendation for the temporary modification/postponement of routine surveillance of asymptomatic cancer survivors in an attempt to reduce risk of COVID-19 transmission through in-person health encounters.

Follow-up care of cancer survivors has been severely impacted by healthcare workforce and resource constraints imposed by the pandemic. Follow-up appointments have been significantly delayed or cancelled, and in-person healthcare interactions have largely been replaced by telehealth as treatment, and follow-up plans have required modification. Some healthcare providers have observed increased anxiety and distress among survivors in relation to telehealth. Many barriers to adopting telehealth have been highlighted including the accessibility of technology, reimbursement practices and privacy and confidentiality concerns. Vast disparities exist in how patient needs are being triaged, and there is limited information regarding how physical examination requirements are being met. Priorities for further survivorship research and practice have been highlighted in triaging the needs of cancer survivors, tele-survivorship care and adopting alternative models of care.

Immunocompromised paediatric cancer survivors have increased susceptibility to COVID-19 infection. In response to this increased risk, some of these children have found themselves confined to their homes where they have limited access to physical activity. This may result in a range of adverse health outcomes and may detrimentally impact the psychosocial wellbeing of survivors. Health support is being provided to such families via instant messaging applications.

Patients with cancer and cancer survivors are a population at increased risk of acquiring COVID-19. Although current evidence regarding the association between cancer and COVID-19 remains inconclusive, increased attention is required to protect patients with cancer and cancer survivors. 
Table 1 (continued)

\begin{tabular}{|c|c|c|c|c|}
\hline Study & Study aim & $\begin{array}{l}\text { Type of } \\
\text { publication }\end{array}$ & Country of origin & Relevant findings and conclusions \\
\hline Dietz et al. [8] & $\begin{array}{l}\text { To categorize breast cancer } \\
\text { (BC) patients in priority } \\
\text { levels based on urgency of } \\
\text { care and provide } \\
\text { treatment } \\
\text { recommendations for } \\
\text { each level during the }\end{array}$ & Expert opinion & USA & $\begin{array}{l}\text { Breast cancer patients have been ranked based on a "Priority" } \\
\text { classification system that accounts for the severity of } \\
\text { individual patient's condition. Through this system, } \\
\text { routine follow-up, survivorship visits and screening have } \\
\text { been placed in Priority C as it is considered that such } \\
\text { patients may be seen remotely or may have follow-up } \\
\text { postponed. }\end{array}$ \\
\hline
\end{tabular}
[19]

Huang et al. [20] survivorship care during the COVID-19 pandemic.

Jhaveri et al. [21] To illustrate the rapid transition of a cancer survivorship program to telehealth and its impact on cancer survivors during COVID-19.

Koczwara [11]

\section{To explore cancer} survivorship care at the time of the COVID-19 pandemic.
To examine head and neck

Expert opinion Canada

\section{Literature \\ review}

USA

Expert Opinion USA

Expert opinion Australia

Liang et al. [22]
The COVID-19 pandemic has necessitated the transition of many module-specific clinic operations (including introductory sessions, sexual rehabilitation clinic, exercise, nutrition, ADT-related group education, pelvic floor physiotherapy and counselling services) to be delivered through online platforms. However, some elements of the clinic operations (related to sexual rehabilitation clinic, exercise and pelvic floor therapy) have been temporarily suspended, postponed or removed. While patients have adapted to the changes in healthcare delivery, challenges remain in extending virtual health care based on different virtual health platform subscriptions and regulatory requirements and in delivering care to patients in remote areas.

Delays in treatment for cancer survivors during the COVID-19 pandemic may detrimentally impact their mental health and quality of life for survivor resulting in downstream burdens on the healthcare system. Telemedicine is recommended as a potential solution that may reduce future burden on the healthcare system and patient wellbeing.

Cancer survivors have demonstrated increased supportive care needs during the pandemic. The interdisciplinary cancer survivorship wellness group program was converted to a telehealth format rapidly and was modified, by necessity, to deliver support in physical activity, nutritional support, sleep, stress management and spiritual wellness remotely. Survivors note that the new medium removed some barriers to access but also noted an increased need for behavioural health and social support in the midst of COVID-19.

In the context of the global pandemic, survivorship care planning visits have been cancelled/postponed despite the fact that cancer survivors experience greater psychological distress and difficulties managing physical symptoms and comorbidities compared to the general population. Survivors may not be able to adhere to lifestyle recommendations due to COVID-19 restrictions and are more likely to be economically vulnerable. Again, cancer survivors appear to be getting lost in transition. The pandemic has required some face-to-face interactions to be transitioned online; however, physical examinations are not possible and exercise programs previously delivered by physiotherapists and other healthcare professionals have been adapted for self-administration.

Cancer patients and cancer survivors with current or past cancer diagnosis may be at increased risk of significant morbidity (including ICU admission, requirement for invasive ventilation or death) compared to patients without cancer. As such, the necessity of any interventions must be weighed against risk of inadvertent nosocomial COVID-19 exposure. Strategies that may be employed to 
Table 1 (continued)

\begin{tabular}{lll}
\hline Study $\quad$ Study aim & $\begin{array}{l}\text { Type of } \\
\text { publication }\end{array}$ & Country of origin Relevant findings and conclusions
\end{tabular}

Nekhlyudov et al. To describe how the [13]

$\mathrm{Ng}$ et al. [23]

Nilsen et al. [24]

Hamlish [25]

Young et al. [27]

To examine the psychosocia consequences of COVID-19 on people with cancer and their caregivers and potential solutions to deal with identified challenges.

Zhou et al. [28] To examine the impact of the COVID-19 pandemic on

Retrospective cohort study protect such patients include: (1) postponing adjuvant chemotherapy or elective surgery for stable cancer (2) stronger personal protection recommendations for cancer patients and survivors and (3) concentrated surveillance and treatment for infected patients.
Expert opinion USA/Canada

Prospective
cohort study

cancer survivors and healthy controls during the COVID-19 pandemic.

survivorship care team to

care

COVID-19 pandemic.

To examine patient-reported Descriptive treatment delays in breast study available for oral cancer survivors during the COVID-19 pandemic.
USA

USA

Sri Lanka

Expert opinion (2) by a multidisciplinary

The COVID-19 pandemic may disproportionately impact the physical and psychosocial wellbeing of cancer survivors. Physical distancing from places of worship, community organizations, families and social circles may detrimentally impact the wellbeing of survivors who are dependent on such networks. Survivors may experience increased anxiety due to redirection of or closure of healthcare resources, added responsibilities at home and financial consequences of COVID-19. Cancellation, postponement, scale back or modification of healthcare services including follow-up visits and surveillance imaging may increase anxiety for cancer survivors.

Cancer survivors were found to report greater catastrophising and health anxiety (related to perceived likelihood and severity of becoming ill) with respect to COVID-19 but less psychological distress compared to healthy controls. Approximately 1 in 8 individuals in both groups reported borderline-to-clinical levels of anxiety while 1 in 7 cancer survivors and 1 in 9 healthy controls indicated borderline-to-clinical levels of depressive symptoms.

The COVID-19 pandemic has resulted in drastic changes to the delivery of survivorship care components with most care being delivered remotely unless absolutely necessary. Survivors and healthcare professional have been required to rapidly adapt to new challenges associated with delivering and receiving survivorship care including nutritional education, speech-language pathology and physical therapy.

Delays in cancer care and treatment were reported by $44 \%$ of cancer survivors across all aspects of cancer care and treatment. The results indicate that the impact of COVID-19 is widespread across cancer care and gaps in disaster preparedness may result in poor outcomes for cancer survivors.

The COVID-19 lockdown restrictions detrimentally impacted availability and accessibility of routine healthcare services for cancer patients. Oral cancer survivors experience problems related to pain and difficulty swallowing and accessing routine treatment and acquiring required medication became a significant concern. Phone-based supportive care delivered by healthcare professional and tailored to the needs of oral cancer survivors appeared to be useful and simple in ensuring patients receive timely care and treatment.

In the context of the COVID-19 pandemic, cancer survivors may have difficulty interpreting whether experienced symptoms are the result of COVID-19 or cancer recurrence and may be unsure whether to report them to their healthcare provider.

Reduced insomnia severity was observed among young adult cancer survivors following the widespread institution of quarantine restrictions due to the COVID-19 pandemic and 
Table 1 (continued)

\begin{tabular}{|c|c|c|c|c|}
\hline Study & Study aim & $\begin{array}{l}\text { Type of } \\
\text { publication }\end{array}$ & Country of origin & Relevant findings and conclusions \\
\hline & $\begin{array}{l}\text { sleep in young adult } \\
\text { cancer survivors. }\end{array}$ & & & $\begin{array}{l}\text { the proportion of individuals demonstrating increased } \\
\text { likelihood of insomnia disorder was lower following } \\
\text { implementation of COVID-19 restrictions. While poor } \\
\text { sleep has been partially attributed for increased levels of } \\
\text { anxiety and depression during the lockdown, these results } \\
\text { highlight the need to consider other factors contributing to } \\
\text { better sleep and increased anxiety and depression during } \\
\text { the pandemic. }\end{array}$ \\
\hline
\end{tabular}

\section{Financial wellbeing}

The final category includes articles that comment on the economic consequences of the pandemic on cancer survivors. Baddour et al. [15] notes that the pandemic and its economic consequences may disproportionately impact cancer survivors and their overall health-related quality of life and mortality. Boakye et al. [16], Nekhlyudov et al. [13] and Koczwara [11] indicate that cancer survivors may be more economically vulnerable and face greater financial hardship during the COVID19 pandemic.

\section{Discussion}

This is the first systematic review of the available literature regarding the wide-ranging impact of the COVID-19 pandemic on cancer survivors. As this review has been conducted as a living rapid review, it will be updated regularly as new and relevant literature on the subject becomes available. Despite the fact that a combination of terms were employed as part of the search strategy and the reference lists of appropriate articles were hand searched, a very limited amount of relevant literature published till August 27, 2020 was found. Furthermore, most of the selected articles (63\%) depend on expert opinions, presented in various forms, that explore specific aspects of the impact of the COVID-19 pandemic on cancer survivors. Very few of the selected papers expressly examine the potential wide-ranging impacts of the COVID-19 pandemic in any great detail.

In spite of the current lack of extensive and definitive research in the field, the articles identified through this rapid review provide preliminary information regarding potential impacts of the COVID-19 pandemic on cancer survivors. Foremost, the available literature highlights drastic changes to survivorship care services accessible to survivors and the medium by which they are delivered during the pandemic. Survivors have been subject to significant delays and cancellations of follow-up appointments and the rapid transition of in-person healthcare interactions to telehealth in resource settings that permit its widespread implementation [7, 11, 19, 21, 24-26]. As such, the pandemic has affected the availability and accessibility of routine healthcare services. Particularly, physical examinations and some elements of survivorship care (e.g. exercise programs) cannot be effectively delivered remotely and have been temporarily suspended, postponed or removed from survivorship care plans [19, 21, 24]. As efforts are made to reduce risk of nosocomial COVID19 transmission, significant changes have been made to the normal standard of care (in terms of frequency, types of care and mode of delivery) that some survivors have grown to depend on. Any changes to a trusted source of care, which may have previously been perceived as a source of confidence and stability, may become the source of increased distress and anxiety.

In addition, the current literature indicates that the COVID19 pandemic may detrimentally impact the psychosocial and physical wellbeing of cancer survivors. Preliminary research in the field indicates that cancer patients and survivors may have increased susceptibility to COVID-19 infection [18, 22] and as a result, the necessity of interventions must be weighed against the potential risk of inadvertent COVID-19 exposure [18]. In an attempt to protect cancer survivors and due to the redirection of healthcare resources, cancer surveillance and other elements of survivorship care have been postponed or cancelled [7, 11, 19, 21, 24-26]. This may result in increased anxiety, diminished quality of life and delayed diagnosis of any disease recurrence or secondary malignancies [16]. Available articles also highlight that increased anxiety and distress has been observed among some survivors with respect to accessing telehealth services and doubts related to the quality of care available through the remote medium [7]. Strict social distancing restrictions across jurisdictions have also reduced access to social and support networks that some survivors may have previously depended upon for physical and psychosocial support [13]. Preliminary research also indicates that cancer survivors are more prone to catastrophising and health anxiety in relation to COVID-19 compared to healthy controls [23]. Thus, the limited literature currently available provides initial indications that the COVID-19 pandemic may 
detrimentally affect the psychosocial and physical wellbeing of some survivors.

Furthermore, available literature notes that the COVID-19 pandemic may disproportionately impact cancer survivors from an economic standpoint [11, 13, $15,16]$. The pandemic has significantly impacted national economies and has resulted in widespread unemployment. However, some cancer survivors with the option to continue to work during the pandemic may have had to leave work due to immunocompromised status putting them at increased risk of COVID-19 infection. Survivors that have lost their jobs due to the pandemic may be experiencing greater economic burden than the general population due to costs incurred during treatment and recovery $[11,13,16]$. Increased financial hardships may in turn, result of detrimental downstream psychosocial and physical impacts on survivors [15].

Overall, the presently available literature provides preliminary indications of wide-ranging impacts of COVID-19 pandemic on cancer survivors with respect to the requirement to adapt to new means of healthcare delivery as well as their physical, psychosocial and economic wellbeing. The COVID-19 pandemic has left survivors dealing with the consequences of rigorous cancer treatment in the context of new challenges related to social isolation, financial hardship and uncertainty with respect to their ongoing care. The challenges presented by the global pandemic may disproportionately impact the overall wellbeing of cancer survivors, which may result in unintended long-term consequences [29].

\section{Study limitations}

A key limitation of this systematic living rapid review is the fact that study selection was restricted to articles published following the initial detection of SARS-CoV2 given the nature of the subject at hand. Given the limited time frame under consideration and the significant resource demands imposed by the COVID-19 pandemic, limited rigorously designed studies are currently available on the impact of the COVID-19 pandemic on cancer survivors, and the present rapid review had to depend heavily upon various forms of expert opinion articles and preliminary studies highlighting some of the short-term impacts of the pandemic on survivors. As a relatively limited amount of time has elapsed since the beginning of the pandemic, the long-term impacts of the COVID-19 pandemic cannot be examined. The authors acknowledge that due to the limited literature currently available, a critical appraisal of available evidence could not be conducted. However, this is a living review that will be updated frequently as the evidence based develops.

\section{Conclusions}

Currently, limited definitive or conclusive evidence exists regarding the impact of the COVID-19 pandemic on cancer survivors. Overall, the presently available literature provides preliminary indications of wide-ranging impacts of COVID19 pandemic on cancer survivors with respect to the requirement to adapt to new means of healthcare delivery as well as their physical, psychosocial and economic wellbeing. The COVID-19 pandemic has left survivors dealing with the consequences of rigorous cancer treatment in the context of new challenges related to social isolation, financial hardship and uncertainty with respect to their ongoing care. The challenges presented by the global pandemic may disproportionately impact the overall wellbeing of cancer survivors, which may result in unintended long-term consequences [29]. Increased rigorously designed research initiatives are required to elucidate the impact of the pandemic on cancer survivors.

Data availability Not applicable.

\section{Compliance with ethical standards}

Conflict of interest The authors declare that they have no conflicts of interest.

Ethics approval Not applicable.

Consent to participate Not applicable.

Consent for publication Not applicable.

Code availability Not applicable.

\section{References}

1. World Health Organization (2020) COVID-19 situation report-94. World Health Organization. https://www.who.int/docs/defaultsource/coronaviruse/situation-reports/20200423-sitrep-94-covid19.pdf. Accessed 27 July 2020

2. World Health Organization (2020) COVID-19 significantly impacts health services for noncommunicable diseases. World Health Organization. https://www.who.int/news-room/detail/0106-2020-covid-19-significantly-impacts-health-services-fornoncommunicable-diseases. Accessed 27 July 2020

3. Azarpazhooh MR, Morovatdar N, Avan A, Phan TG, Divani AA, Yassi N, Stranges S, Silver B, Biller J, Tokazebani Belasi M, Kazemi Neya S, Khorram B, Frydman A, Nilanont Y, Onorati E, di Napoli M (2020) COVID-19 pandemic and burden of noncommunicable diseases: an ecological study on data of 185 countries: COVID-19 and non-communicable diseases. J Stroke Cerebrovasc Dis 29(9):105089. https://doi.org/10.1016/j. jstrokecerebrovasdis.2020.105089

4. Kutikov A, Weinberg DS, Edelman MJ, Horwitz EM, Uzzo RG, Fisher RI (2020) A war on two fronts: cancer care in the time of COVID-19. Ann Intern Med 172:756-758. https://doi.org/10.7326/ M20-1133 
5. Qian Y, Wu K, Xu H, Bao D, Ran F, Wei W, Cheng T, Huang D, Lin X, Bruera E, Hu D, Wu Y (2020) A survey on physical and mental distress among cancer patients during the COVID-19 epidemic in Wuhan, China. J Palliat Med 23(7):888-889

6. Calvillo-Argüelles O, Abdel-Qadir H, Ky B, Liu JE, Lopez-Mattei JC, Amir E, Thavendiranathan P (2020) Modified routine cardiac imaging surveillance of adult cancer patients and survivors during the COVID-19 pandemic. JACC CardioOncol 2(2):345-349

7. Chan A, Ashbury F, Fitch MI et al (2020) Cancer survivorship care during COVID-19 - perspectives and recommendations from the MASCC survivorship study group. Support Care Cancer 28: 3485-3488

8. Dietz JR, Moran MS, Isakoff SJ et al (2020) Recommendations for prioritization, treatment, and triage of breast cancer patients during the COVID-19 pandemic. The COVID-19 pandemic breast cancer consortium. Breast Cancer Res Treat 181(3):487-497

9. Hanna TP, Evans GA, Booth CM (2020) Cancer, COVID-19 and the precautionary principle: prioritizing treatment during a global pandemic. Nat Rev Clin Oncol 17(5):268-270

10. Salako O, Okunade K, Allsop M et al (2020) Upheaval in cancer care during the COVID-19 outbreak. Ecancermedicalscience 14: ed97

11. Koczwara B (2020) Cancer survivorship care at the time of the COVID-19 pandemic. Med J Aust 213:107-108.e1. https://doi. org $/ 10.5694 / \mathrm{mja} 2.50684$

12. Rosenbaum L (2020) The untold toll- the pandemic's effects on patients without Covid-19. NEJM 382:2368-2371

13. Nekhlyudov L, Duijts S, Hudson SV, Jones JM, Keogh J, Love B, Lustberg M, Smith KC, Tevaarwerk A, Yu X, Feuerstein M (2020) Addressing the needs of cancer survivors during the COVID-19 pandemic. J Cancer Surviv 14:601-606

14. Moher D, Liberati A, Tetzlaff J, Altman DG, The PRISMA Group (2009) Preferred reporting items for systematic reviews and metaanalyses: the PRISMA statement. PLoS Med 6(7):e1000097

15. Baddour K, Kudrick LD, Neopaney A, Sabik LM, Peddada SD, Nilsen ML, Johnson JT, Ferris RL, Mady LJ (2020) Potential impact of the COVID-19 pandemic on financial toxicity in cancer survivors. Head Neck 42(6):1332-1338

16. Boakye EA, Jenkins W, Sharma A (2020) Disproportionate impact of COVID-19 pandemic on head and neck cancer survivors. Head Neck 42(6):1329-1331

17. Cheung AT, Li WH, Ho LL et al (2020) Health support to pediatric cancer survivors and their families during the COVID-19 pandemic. Pediatr Blood Cancer 67. https://doi.org/10.1002/pbc.28441

18. Desai A, Sachdeva S, Parekh T, Desai R (2020) COVID-19 and cancer: lessons from a pooled meta-analysis. JCO Glob Oncol 6: $557-559$
19. Flannigan R, Sundar M, Weller S, Ivanov N, Hu M, Dayan M, Chen C, Wu E, Goldenberg L, Higano CS (2020) Pearls to pivoting a multidisciplinary prostate cancer survivorship program during the COVID-19 pandemic. Eur Urol Oncol 3(4):397-399

20. Huang VW, Imam SA, Nguyen SA (2020) Head and neck survivorship care in the times of the SARS-CoV-2 pandemic. Head Neck 42:1664-1667

21. Jhaveri K, Cohen JA, Barulich M, Levin AO, Goyal N, Loveday T, Chesney MA, Shumay DM (2020) "Soup cans, brooms, and Zoom: " rapid conversion of a cancer survivorship program to telehealth during COVID-19. Psychooncology 29:1424-1426. https://doi.org/ 10.1002/pon.5473

22. Liang W, Guan W, Chen R, Wang W, Li J, Xu K, Li C, Ai Q, Lu W, Liang H, Li S, He J (2020) Cancer patients in SARS-CoV-2 infection: a nationwide analysis in China. Lancet Oncol 21(3):335337

23. Ng D, Chan F, Barry TJ et al (2020) Psychological distress during the 2019 coronavirus disease (COVID-19) pandemic among cancer survivors and healthy controls. Psychooncology 29:1380-1383. https://doi.org/10.1002/pon.5437

24. Nilsen ML, Clump DA, Kubik M et al (2020) Prevision of multidisciplinary head and neck cancer survivorship care during the 2019 novel coronavirus pandemic. Head Neck 42:1668-1673

25. Papautsky EL, Hamlish T (2020) Patient-reported treatment delays in breast cancer care during the COVID-19 pandemic. Breast Cancer Res Treat 184:249-254. https://doi.org/10.1007/s10549020-05828-7

26. Ratnasekera N, Perera I, Kandapolaarachchige P, Surendra G, Dantanarayana A (2020) Supportive care for oral cancer survivors in Covid-19 lock down. Psychooncology 29:1409-1411. https:// doi.org/10.1002/pon.5463

27. Young AM, Ashbury FD, Schapira L, Scotté F, Ripamonti CI, Olver IN (2020) Uncertainty upon uncertainty: supportive care for cancer and COVID-19. Support Care Cancer 28:4001-4004

28. Zhou ES, Michaud AL, Owens J, Recklitis CJ (2020) Sleep in young adult cancer survivors during the COVID-19 pandemic. J Clin Sleep Med 16:1991. https://doi.org/10.5664/jcsm.8716

29. Battisti NM, Mislang AR, Cooper L et al (2020) Adapting care for older cancer patients during the COVID-19 pandemic: recommendations from the International Society of Geriatric Oncology (SIOG) COVID-19 Working Group. J Geriatr Oncol 11:1190 1198. https://doi.org/10.1016/j.jgo.2020.07.008

Publisher's note Springer Nature remains neutral with regard to jurisdictional claims in published maps and institutional affiliations. 